\title{
Critical Factors for a Successful Astronomical Research Program in a Developing Country
}

\author{
John B. Hearnshaw
}

Department of Physics and Astronomy, University of Canterbury, Christchurch, New Zealand. e-mail j.hearnshaw@phys.canterbury.ac.nz

\begin{abstract}
.
I discuss the critical conditions for undertaking a successful research program in a developing country. There are many important factors, all or most of which have to be satisfied: funding, library holdings, computing access, Internet access (e-mail, WWW, ftp, telnet), collaboration with astronomers in developed countries, provision of proper offices for staff, supply of graduate students, access to travel for conferences, ability to publish in international journals, critical mass of researchers, access to a telescope (for observational astronomers), support from and interaction with national electronics, optics and precision engineering industries, a scientific culture backed by a national scientific academy, and lack of inter-institutional rivalry. I make a list of a total of 15 key factors and rank them in order of importance, and discuss the use of an astronomical research index (ARI) suitable for measuring the research potential of a given country or institution.

I also discuss whether astronomers in developing countries in principle fare better in a university or in the environment of a government national observatory or research institution, and topics such as the effect of the cost of page charges and journal subscriptions on developing countries. Finally I present some statistics on astronomy in developing countries and relate the numbers of astronomers to the size of the economy and population in each country.
\end{abstract}

\section{Introduction}

The purpose of this article is to consider those factors which contribute towards and are essential for a successful astronomical research program in any country, especially those countries which are developing economically. I have identified fifteen key factors which contribute to the viability of research. Most of them are essential if astronomical research is to be successfully pursued.

Adequate funding is obviously a key to most things, and although I identify this as the single most important factor, and although it is involved to some extent in all the factors that I have identified, it is too simple to assert that money is the only important thing for research. For example, the prevailing culture of scientific enquiry and the interactions with high-tech industry and with the teaching profession also play important roles. 
Ideally, having identified fifteen separate factors, an astronomical research index (ARI) can be defined which measures the research potential of any given country (or institution). How the ARI should be defined in terms of the fifteen factors $n_{1} \ldots n_{15}$ requires some care, so as to take account of two things: first, the relative importance of all fifteen factors is not the same - some (such as funding, computers and library holdings) feature far more prominently than others. Secondly, whereas a high score in all factors is conducive to research being successfully undertaken, a low score in just one of them may make effective research almost impossible, irrespective of the values of the other factors. This being the case, it is clear that the ARI must be a non-linear combination of the factors. In section 4 a logarithmic expression is proposed that takes this into account.

\section{Fifteen Key Factors for a Successful Research Program}

I identify fifteen factors that underpin successful research. They are introduced in what I consider to be their relative order of importance. The list is of course subjective, and to some extent the relevant factors are inter-related, given that the boundaries separating them are not always clear-cut.

\section{Access to direct funding}

Although most things in astronomy depend on funding, I consider here direct funding for astronomy, either in the form of individual research grants, or as institutional funding to observatories or to university astronomy departments, both for their operations and for particular research projects.

\section{Access to computers}

Every astronomer today needs a computer for data reduction and analysis. This could be a PC, a mainframe computer in a local area network, or sometimes a powerful supercomputer, possibly in a national facility. In computer-rich nations, several computing options will be available 24 hours a day to all researchers, and many individuals will carry laptops and have private computers at home. Computers will be replaced every 3 or 4 years on average. In a developing country, one or two older PCs might be shared in a university department. In this section I do not include computers for Internet access - this is sufficiently important for a separate category (see No. 10 below).

\section{Access to an astronomical library}

A good library is clearly essential if research of international standard is to be done. The major astronomical journals must all be subscribed to, and holdings need to go back several decades if research is not to be impaired. Journals must be available to all researchers, and be located at or near the place of their work. In addition to journals, a reference library, a good collection of relevant conference proceedings and a collection of review articles (e.g. Annual Review of Astronomy \& Astrophysics) and of books (including text-books) are also required. Good libraries will also have a collection of observatory publications. The ability to browse in libraries is 
important (access to the shelves is required), and prompt receipt of about a dozen major journals is also important.

Good libraries help to ensure that research topics are relevant and topical in a global context, and that proper standards of scholarship are maintained, including awareness of what has been accomplished by others in the past, and what is important now and for the future. Unfortunately, the cost of maintaining libraries is prohibitive for many developing countries. Not only are the journal subscriptions very high, but the true cost can be at least double the subscription, given the need to provide shelf space and a librarian. High inflation and the fact that subscriptions are usually quoted in U.S. dollars render many journals unobtainable in the developing world.

\section{The provision of office space}

Astronomers need to be housed in proper offices in which there is the normal office furniture, heating, lighting and storage space for books and papers. Offices need to be clustered together, so as to promote interactions between individuals. And offices need to be dedicated to one or at most a few individuals, and access should be provided on a continuous basis, seven days a week, and 24 hours a day.

\section{Having a critical mass of researchers}

Most astronomers learn their trade by interacting with others. Few can work in isolation. Isaac Newton certainly did for much of his scientific career, but even he needed the data of John Flamsteed in order to verify his theories! Today, astronomy is a vast web of knowledge, information, technology and know-how, which no individual could conceivably master unassisted. I consider a minimum critical mass of full-time active researchers interacting on a daily basis to be about four. Doubling this number to eight would however far more than double output.

\section{Access to a telescope (or to raw data)}

Telescope access is vital for observational astronomers, or for those whose research involves data analysis or its interpretation. Even theoretical astronomers need to have data to input into empirical models, and only the most abstract theoreticians can eschew data altogether. For many observational astronomers, data comes from telescopes; astronomers in many developing countries would consider that a telescope on home soil would not only greatly facilitate their research, but also act as a symbol of prestige. Unfortunately, not all telescopes in developing countries are adequately maintained, operated or equipped with instruments and detectors, so the mere presence of a telescope and willing astronomers is no guarantee of a flow of good-quality data. Today, in optical astronomy, a CCD detector is increasingly seen as an indispensable accessory to any telescope, yet the cost and necessary infrastructure (with liquid-nitrogen cryogenics and vacuum technology) are beyond the means of many developing countries.

If telescopes and associated instruments are not provided by the institution where astronomers are employed, then they need to be provided in a national or international facility that provides access to astronomers 
from developing countries. The outstanding example is Cerro Tololo Interamerican Observatory, that in principle provides access to South-American astronomers. Most astronomers in developing countries are not so fortunate.

\section{The presence of a teaching program, and the consequent supply} of graduate students

Graduate students are a huge bonus for any research team, and a university astronomy department with a ratio of full-time graduate students doing research to tenured academics of, say, two or three to one can enjoy the contribution from often talented young individuals, who are eager to work long hours for low pay and to provide ideas and enthusiasm. Governmentfunded observatories do not always have access to this resource, much to their detriment.

A flow of new graduate students every year in turn probably requires an active teaching program, especially at undergraduate levels, and which encompasses aspects of the research interests of the department. In universities the link between teaching and research is often asserted. Certainly some teaching broadens the research perspective of tenured lecturers, and undergraduate teaching stimulates interest in astronomy, thereby promoting the enrolment of graduate students and the willingness to consider astronomy as a career.

\section{Collaborations with astronomers from developed countries}

Astronomy is a global science, and collaborations provide another way of bringing in expertise, know-how, high-tech equipment and sound scientific judgement to a research project. Astronomers in developing countries, if they work in isolation, may not only lack the resources, but also the ideas to mount a successful research program. Collaboration is an ideal way of surmounting this. Those in astronomically favoured locations, such as Chile, certainly have a significant advantage. Those in moist tropical climates or inclement high-latitude ones may have less to offer, but some developing countries can exchange scientific benefits for cultural ones. In New Zealand we have a successful CCD microlensing project called MOA undertaken jointly between astronomers in New Zealand and Japan. Our observing site at Mt John is certainly not the world's best, although it offers some unique advantages (southern latitude, unique longitude); but in addition we offer our Japanese visitors a landscape and a lifestyle that contrast with and nicely complement those in Japan.

\section{Travel to conferences}

Travel to conferences is very much a part of the life of the scientist in today's world. The IAU General Assembly is testimony to that. Many astronomers in developed countries regularly participate in two or three international conferences annually. Conferences provide a further opportunity for personal interactions and exchange of ideas, vital for science to flourish in a modern environment. In the nineteenth century, conferences were largely conducted nationally through the meetings of bodies such as the Royal Astronomical Society, the Astronomische Gesellschaft and the 
Académie des Sciences (to mention only three). Astronomers were also pioneers in promoting international conferences, and the meetings of the Astrographic Congress (that organized the Carte du Ciel) from 1887 and of the International Solar Union (in the early 20th century) were important predecessors to the foundation of the International Astronomical Union in 1920. Today there can easily be 50 or 60 astronomical conferences held around the world in any year. Participation is an important part of an astronomer's work, and conference papers may well constitute a majority of the papers published by any individual.

Participation at conferences on a regular annual basis requires not only funding, but also a lack of imposed travel restrictions and an absence of political interference in the scientific process in any country (both in the participants' home countries and in that of the conference host).

Countries like New Zealand are a long way from anywhere. This means that New Zealand astronomers only rarely host international conferences (few would come) and moreover, we must pay far more than many others to travel. Fortunately we are often able to make one or two international trips annually, usually to distant places.

10. Good communications: internet access, fax, telephones, mail Communications are vital for astronomy. The Internet, including e-mail, $\mathrm{ftp}$, telnet, and the WWW, is used by most astronomers on a daily basis. But astronomers also need fax machines, telephones in every office, and a reliable mail service. In developed countries, all these are taken for granted, but they may not exist at all in the developing world. The organizer of this Special Session, Alan Batten, has told me about the problems he had in communicating with home base in Canada from Nigeria. It is not always simple! Collaborations, data access and information all flow from good information technology. Some see the Internet as an alternative to more expensive libraries and telescopes; I doubt that it can ever be a complete substitute in the long run, but many developing countries may see the Internet as a possible replacement for libraries and telescopes in the interim.

\section{The ability to publish in international journals}

The whole question of the availability of the leading scientific journals for astronomers from developing countries to publish their papers is a thorny issue. For many, the page charges of the leading three American journals (Astrophys. J, Astron. J, PASP) and of Astron. \& Astrophys. in Europe prohibit publication unless one has a collaborator as co-author in respectively North America or Europe. Some of these journals may waive page charges for authors from developing countries at the editor's discretion, which is a welcome but not widely invoked practice. Admittedly a few leading journals have no page charges (notably MNRAS), but high subscription costs then limit dissemination to libraries in the developing world.

Personally I believe that high but differentiated page charges (i.e. less or none for authors from developing countries) and very low subscriptions are 
the way forward, because then astronomers might curtail the huge volume of papers published, which in many cases few have time to read. The number of papers is at present driven by the "publish or perish" syndrome in the United States and other developed countries. We have a crisis in scientific publishing as a result. Electronic journals may force a solution on us, but if electronic publishing leads to lower costs to authors, it will not serve any great purpose, as lower costs will result in yet greater volumes of material that is read by few or even no-one. The solution is probably a very high cost to authors in the developed world for refereed electronic publications, publication costs being reduced or waived in the developing world, dissemination of articles via the Internet or CD-ROM, and low cost or free subscriptions. This would force astronomers to publish less, and enable scientists in the developing world better access to the literature. A much greater use of unrefereed web sites would be made to exchange information between specialists working in particular fields.

\section{Employment opportunities}

Many students are turned away from astronomy because they perceive there to be poor employment opportunities. This is especially true in developing countries, because astronomy is often regarded as not an essential part of national development goals. I note that the South African government's backing for SALT is a refreshing exception to this rule (Martinez 2001).

Students need identifiable career paths before them. That means a vigorous graduate program followed by a regular supply of postdoctoral fellowships, and at least occasional opportunities for tenured positions at universities and government observatories.

Postdoctoral positions are often a weak link, and this was certainly true in New Zealand until the mid-1990s. Fortunately both the government and the universities have heeded this weakness, and in recent years we have benefitted at Canterbury from a ratio of tenured astronomers to postdocs of about $1: 1$.

\section{Lack of inter-institutional rivalry}

Some may be surprised that I include this item; others who have experienced the destructive effects of rivalry between two institutions in a country, each vying for limited resources, will understand how damaging this is for the longer-term prospects of astronomy. Often this rivalry is between government-funded observatories and astronomy departments in the universities. Both the United Kingdom and New Zealand have had such rivalries in the past, both to their respective detriment. Fortunately these events are now history. I know that Australia has had them too, and I am aware of other serious cases elsewhere. In reality they are probably very common, and arise from the different ways in which universities and government-funded research institutions allocate their resources and set their priorities. Such squabbles can occur anywhere, and can cripple a developing country's effort in astronomy. Possibly the smaller the country and the scarcer the resources, often the more intense are the battles waged to assume power and control of whatever resources that can be allocated. 


\section{Opportunities for interactions with high-tech industry}

Astronomy is an expensive and high-tech science. For example, optical astronomers may well decide to build telescopes and instruments which will require well-developed optical, mechanical and electronic engineering capabilities as well as sophisticated computer control software. Partnerships between astronomers (whether in universities or government observatories) rarely occur in the developing world (such industries are generally absent). One only has to look at the enormous benefit to astronomy of the commercial and military development of CCD detectors and of infrared arrays to realize how astronomical progress is tied to developments in electronics, which are in turn driven by commercial and military interests in the developed world.

But on a much smaller scale, such interactions can and do take place outside the G7 countries of North America, Europe and Japan. For example, in New Zealand at the University of Canterbury, we have had a marvellous relationship over 25 years with optical design and fabrication engineers based in the former DSIR Physics and Engineering Laboratory (now operated as a commercial company, IRL). This relationship continued with the individuals concerned after their retirement. It has enabled us to build the 1-m telescope and most of the instruments at Mt John University Observatory.

\section{The presence of a national scientific culture}

For astronomy to flourish, science as a whole must flourish, and that needs a scientific culture to have developed. This may come from having a national academy at the helm; in New Zealand we have the Royal Society of N.Z., with an academy of fellows which can advise the government on science policy. However a scientific culture or respect for science probably starts much lower down, in the schools. In Japan, Singapore and South Korea, science is given a high priority in the schools, perhaps because science teaching is a well-respected profession. Students then want to be scientists (including astronomers) from an early age. The acceptance of astronomy as a taxpayer-funded endeavour requires such a climate for its national acceptance.

\section{Can the Potential for Astronomical Research be Measured? - the Astronomy Research Index (ARI)}

Devising an astronomical research index (ARI) as a measure of the potential of a country, or even an institution, to undertake astronomical research is a useful way of intercomparing countries or institutions within a country, and of identifying any weaknesses. My approach is as follows.

For each of the fifteen factors (in the order presented in section 2), a numerical quantity $n_{i}$ is determined on a linear scale from 0 to 10 . Zero represents a complete absence of that factor, while 10 represents the current best in the world (e.g. as represented by the United States). A value of about 5 for any factor would indicate a marginal value for mounting a successful astronomical 
research program. The assigning of values to these 15 factors is rather arbitrary, but generally developed countries will record 8,9 or 10 for all of them.

Next, I have assigned weights $\left(w_{i}\right)$ to the fifteen parameters to reflect the different level of importance they have for research. For simplicity, my weights are unity for $n_{1}$ to $n_{4}$ inclusive, $w_{i}=\frac{2}{3}$ for $n_{5}$ to $n_{11}$, and $w_{i}=\frac{1}{3}$ for $n_{12}$ to $n_{15}$.

I note that a simple weighted mean of the $n_{i}$ parameters would not be a meaningful ARI, because in practice a low value for any one parameter can completely nullify the entire research effort. For this reason, I define the index logarithmically:

$$
\mathrm{ARI}=\Sigma w_{i} \log n_{i}
$$

In the United States, where by definition the $n_{i}$ values are all 10, one obtains ARI(US) $=10.0$. In practice I consider ARI $\sim 8.0$ as the minimum for a viable astronomical research program. Because this is a logarithmic scale, that represents about $1 \%$ of the resources being allocated to astronomy per astronomer that is allocated in the U.S. If, for example, all factors are individually $n_{i}=5$, then $\mathrm{ARI}=7.0$, which, taken as a whole, is below my approximate critical value of the index for undertaking research successfully.

Table 1. Table of factors conducive to research. The final column gives values of the factors for New Zealand astronomy at the University of Canterbury

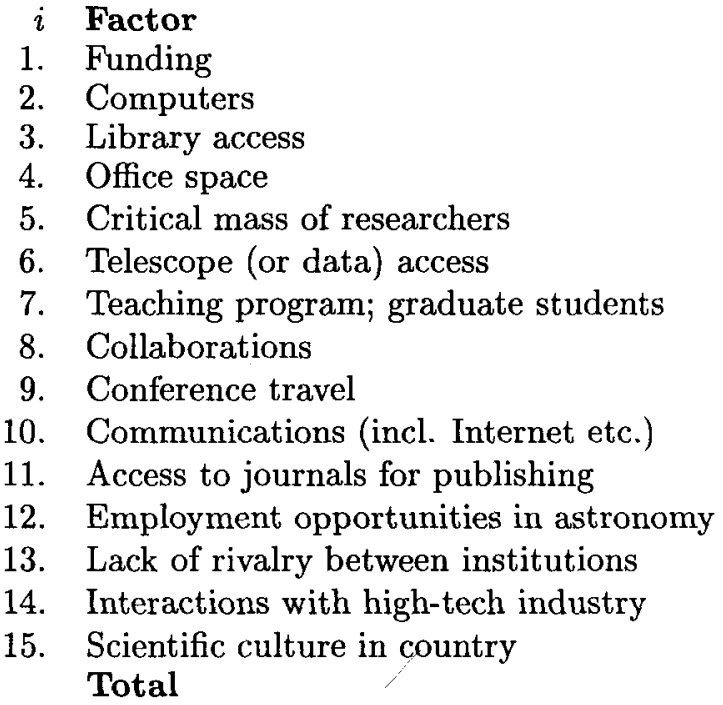

$i$ Factor

1. Funding

Computers

3. Library access

4. Office space

5. Critical mass of researchers

6. Telescope (or data) access

7. Teaching program; graduate students

8. Collaborations

9. Conference travel

10. Communications (incl. Internet etc.)

11. Access to journals for publishing

13. Lack of rivalry between institutions

14. Interactions with high-tech industry Total

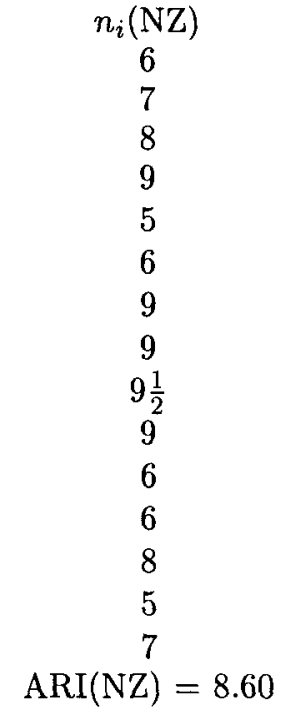

Table 1 lists the factors and their weights and gives, as an example, the estimated figures for the University of Canterbury, which is the principal place for astronomical research in New Zealand. Here ARI $(\mathrm{NZ})=8.60$, and the weak links (with $n_{i}=5$ ) are barely a critical mass of researchers (only four tenured astronomy academics), and few interactions with local high-tech industry. However, our strong points $\left(n_{i} \sim 9\right)$ are good Internet and communications access; 
good opportunities for collaboration (e.g. the MOA project, SALT); good provision of office space for astronomers; a good supply of talented graduate students and excellent opportunities for international travel.

\section{Some Statistics on Astronomers, Demography and Economic Development}

The IAU comprises 61 member countries with 8223 astronomers as individual IAU members (IAU Inf. Bull 82, 14 (1998)). In addition, there are 105 IAU members in a further 21 countries that do not adhere to the union. These statistics include the Central American Association for Astronomy, an association of six countries treated as a single member state. Of these 82 countries in total, which are either IAU member countries, or contain IAU individual members, all but five are also members of the International Monetary Fund (these five are China Taipei, the Vatican City State, Yugoslavia, North Korea and Cuba). I have used IMF data on the state of economic development in the remaining 77 countries; a useful single parameter is the IMF quota (in units of special drawing rights, SDR), which is based on national income, monetary reserves, trade balance and other economic indicators. IMF quotas have been taken from the Europa World Year Book (1998), as have total populations in each country (generally valid for about 1997). Table 2 lists these data, as well as the numbers of IAU members residing in each country.

Figure 1 shows there is a strong correlation between the IMF quota and the number of astronomers in a country. This supports the view that economic strength is essential for astronomical research. More instructive, however, is to plot the IAU astronomers/million of population against the IMF quota/million, as displayed in Figure 2. The latter is a measure of true wealth per head of population. Switzerland, on this basis, is the wealthiest country, while Belgium, Iceland, Saudi Arabia and Norway (in that order) are close behind. There is still a reasonably good correlation between astronomers/million and per capita wealth, though a few countries stand out. Estonia is a developing country, but the only nation with more than 14 astronomers/million. Its wealth is only 31.3 SDR units per million. Any value below about 50 units on this scale is what might be termed a developing country. On the other hand, Saudi Arabia ( 0.6 astronomers/million) and Norway (5.0 astronomers/million) evidently have invested few of their oil revenues into astronomy.

Figure 3 looks at more detail at those 44 countries which

(a) have IAU members or are member countries of the IAU,

(b) belong to the IMF,

(c) have a wealth index (SDR/million) less than 50.0 units.

The following comments can be made:

1. Astronomical activity as measured by the number of astronomers, varies from almost zero to some upper limit which increases linearly with wealth. 
IAU members vs IMF quota (SDR)

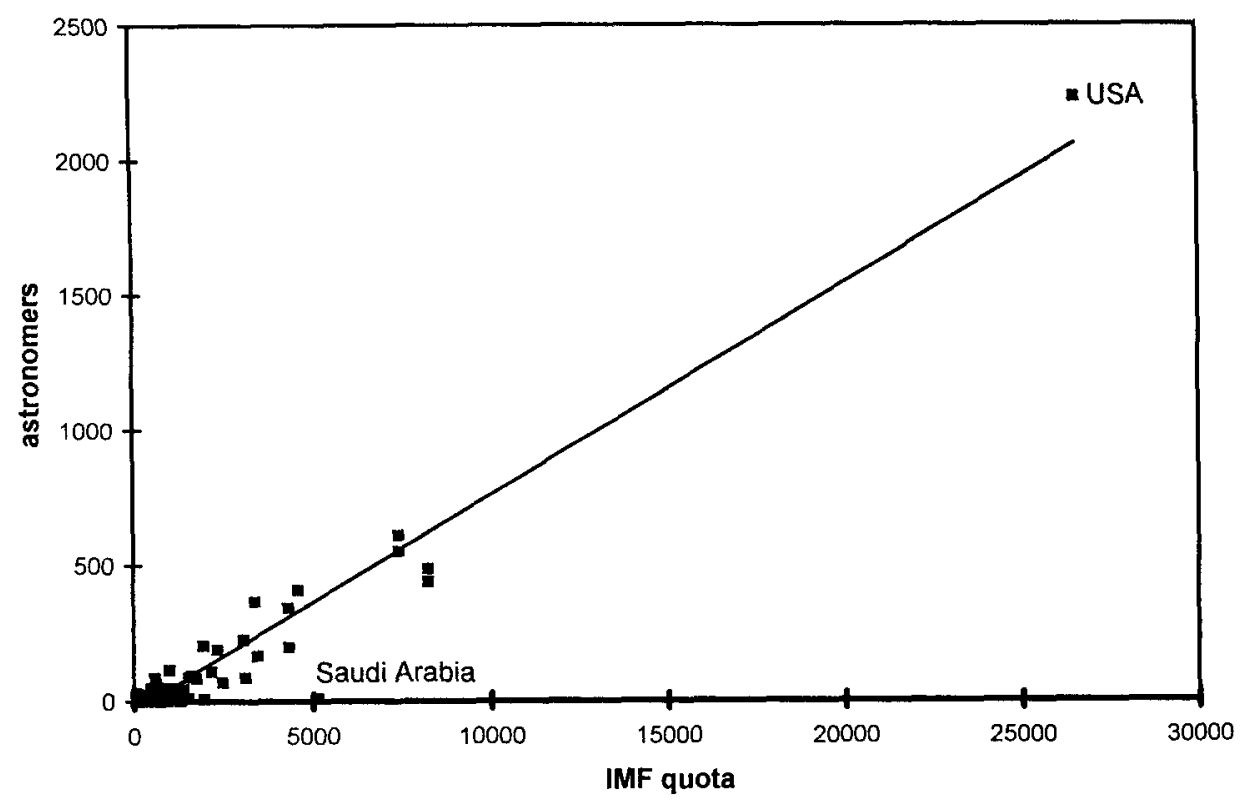

Figure 1. IAU members versus IMF quota (SDR)

Astronomers/million vs wealth

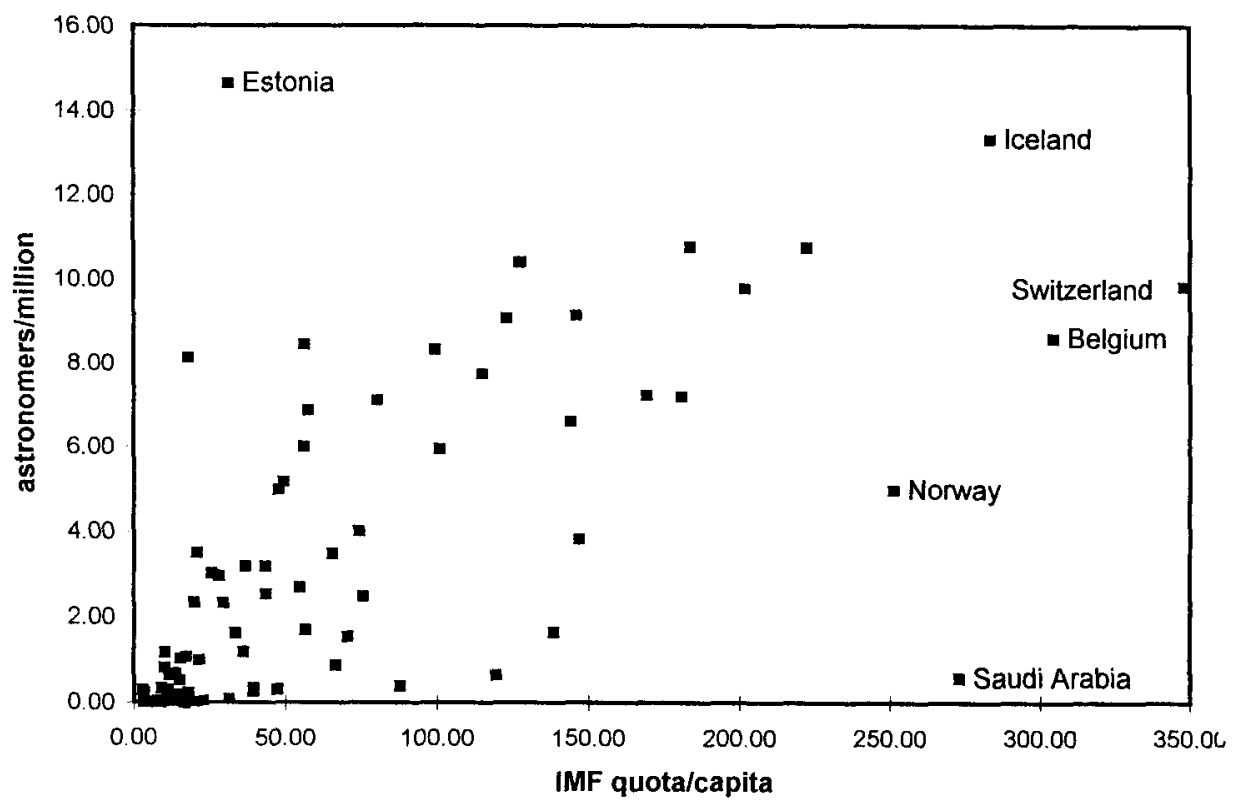

Figure 2. Astronomers/million versus wealth per capita (SDR/million) 
Developing countries: astronomers/million vs wealth/capita

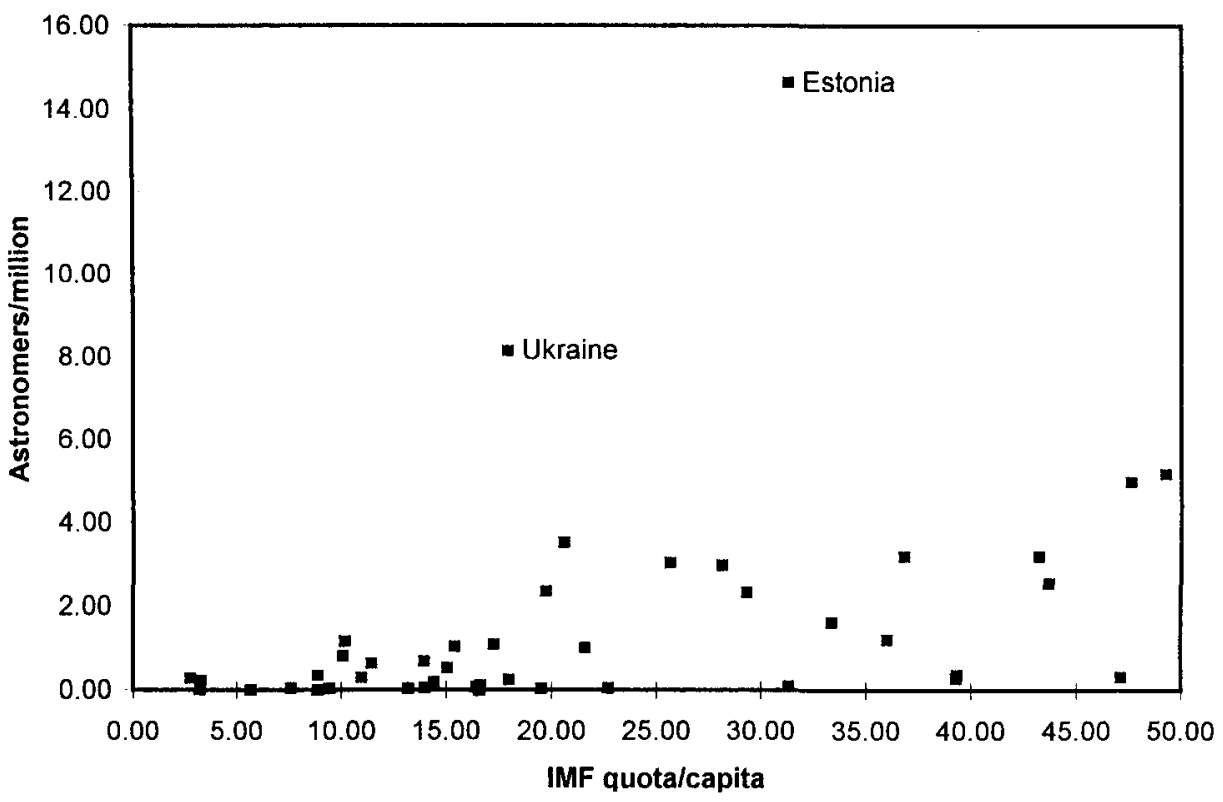

Figure 3. Astronomers/million versus wealth per capita for developing countries

2. Just two developing countries stand out from this trend in having an unusually large number of astronomers/million (Estonia and Ukraine). Probably both these exceptions arose from political circumstances following the break-up of the Soviet Union, rather more than from astronomically enlightened governments, although I congratulate both countries on their good fortune in finding themselves astronomically well-endowed.

The last column of Table 2 gives astronomers/SDR, which is a measure of the number of astronomers per unit of economic activity in a country, and hence is an indicator of the fraction of the available economic resources that have been applied to astronomy. For 44 developing countries this is, on average, 0.066 . This figure compares with 33 developed countries (those with SDR/million of population $>50.0$ ), where astronomers $/ \mathrm{SDR}=0.051$, actually less (though not significantly) than the number in developing countries. This shows that many developing countries are applying similar fractions of their available economic resources to astronomy as developed countries. Nevertheless, that conclusion is biased by the fact that only those developing countries with some astronomical activity are considered in Table 2. Two developing countries (Armenia and Estonia) have values of this index of about 0.46 , much higher than any developed countries. The average value for North America, Western Europe and Japan is 0.064 , and for 59 IAU member countries which are also IMF members, it is 0.074 . 
Table 2: Data on countries pertaining to economy, population and astronomers

\begin{tabular}{|c|c|c|c|c|c|c|}
\hline Country & $\begin{array}{c}\text { IMF quota } \\
\text { (SDR) }\end{array}$ & $\begin{array}{c}\text { Population } \\
\text { (miltions) }\end{array}$ & $\begin{array}{c}\text { Wealth } \\
\text { (SDR/million) }\end{array}$ & IAU members & $\begin{array}{c}\text { astronomers } \\
\text { (/million) }\end{array}$ & $\begin{array}{l}\text { astronomers } \\
(/ \text { SDR }) \times 100\end{array}$ \\
\hline Albania & 35 & 3.2 & 10.94 & 1 & 0.31 & 2.86 \\
\hline Algeria" & 914 & 29.2 & 31.30 & 3 & 0.10 & 0.33 \\
\hline Argentina" & 1537 & 35.2 & 43.66 & 90 & 2.56 & 5.86 \\
\hline Armenia* & 68 & 3.8 & 17.89 & 31 & 8.16 & 45.59 \\
\hline Australia" & 2333 & 18.3 & 127.49 & 191 & 10.44 & 8.19 \\
\hline Austria" & 1188 & 8.1 & 146.67 & 31 & 3.83 & 2.61 \\
\hline Azerbaijan* & 117 & 7.6 & 15.39 & 8 & 1.05 & 6.84 \\
\hline Bahrain & 83 & 0.6 & 138.33 & 1 & 1.67 & 1.20 \\
\hline Belgium* & 3102 & 10.2 & 304.12 & 88 & 8.63 & 2.84 \\
\hline Bolivia* & 126 & 7.6 & 16.58 & 0 & 0.00 & 0.00 \\
\hline Brazil* & 2171 & 155.8 & 13.93 & 109 & 0.70 & 5.02 \\
\hline Buigaria* & 465 & 8.3 & 56.02 & 50 & 6.02 & 10.75 \\
\hline Canada* & 4320 & 30 & 144.00 & 199 & 6.63 & 4.61 \\
\hline Cent. Amer." & 740 & 32.6 & 22.70 & 2 & 0.06 & 0.27 \\
\hline Chile* & 622 & 14.4 & 43.19 & 46 & 3.19 & 7.40 \\
\hline China PR* & 3385 & 1223.9 & 2.77 & 368 & 0.30 & 10.87 \\
\hline China Taipei" & & 21.5 & 0.00 & 23 & 1.07 & \\
\hline Colombia & 561 & 40.2 & 13.96 & 3 & 0.07 & 0.53 \\
\hline Croatia* & 262 & 4.8 & 54.58 & 13 & 2.71 & 4.96 \\
\hline Cuba & & 11 & 0.00 & 5 & 0.45 & \\
\hline Czech Rep." & 590 & 10.3 & 57.28 & 71 & 6.89 & 12.03 \\
\hline Denmark & 1070 & 5.3 & 201.89 & 52 & 9.81 & 4.86 \\
\hline Egypt* & 678 & 59.3 & 11.43 & 39 & 0.66 & 5.75 \\
\hline Estonia" & 47 & 1.5 & 31.33 & 22 & 14.67 & 46.81 \\
\hline Finiand* & 862 & 5.1 & 169.02 & 37 & 7.25 & 4.29 \\
\hline France* & 7415 & 58.4 & 126.97 & 609 & 10.43 & 8.21 \\
\hline Georgia" & 111 & 5.4 & 20.56 & 19 & 3.52 & 17.12 \\
\hline Germany" & 8242 & 81.8 & 100.76 & 488 & 5.97 & 5.92 \\
\hline Greece* & 588 & 10.5 & 56.00 & 89 & 8.48 & 15.14 \\
\hline Hungary & 755 & 10.2 & 74.02 & 41 & 4.02 & 5.43 \\
\hline Iceland" & 85 & 0.3 & 283.33 & 4 & 13.33 & 4.71 \\
\hline India* & 3056 & 936 & 3.26 & 227 & 0.24 & 7.43 \\
\hline Indonesia" & 1498 & 197.5 & 7.58 & 12 & 0.06 & 0.80 \\
\hline $\operatorname{tran}{ }^{*}$ & 1079 & 60.1 & 17.95 & 15 & 0.25 & 1.39 \\
\hline Irac & 865 & 22 & 39.32 & 8 & 0.36 & 0.92 \\
\hline Ireland" & 525 & 3.6 & 145.83 & 33 & 9.17 & 6.29 \\
\hline |srael* & 666 & 5.8 & 114.83 & 45 & 7.76 & 6.76 \\
\hline Italy* & 4591 & 57.4 & 79.98 & 409 & 7.13 & 8.91 \\
\hline Japan* & 8242 & 126.1 & 65.36 & 440 & 3.49 & 5.34 \\
\hline Kazakhstan & 248 & 16.5 & 15.03 & 9 & 0.55 & 3.63 \\
\hline Latvia* & 92 & 2.5 & 36.80 & 8 & 3.20 & 8.70 \\
\hline Lebanon & 146 & 3.1 & 47.10 & 1 & 0.32 & 0.68 \\
\hline Lithuania* & 104 & 3.7 & 28.11 & 11 & 2.97 & 10.58 \\
\hline Malaysia* & 833 & 21.2 & 39.29 & 6 & 0.28 & 0.72 \\
\hline Mauritius & 73 & 1.1 & 66.36 & 1 & 0.91 & 1.37 \\
\hline Mexico* & 1753 & 81.2 & 21.59 & 83 & 1.02 & 4.73 \\
\hline Morocco & 428 & 26.1 & 16.40 & 2 & 0.08 & 0.47 \\
\hline N. Korea & & 21.2 & 0.00 & 20 & 0.94 & \\
\hline Netherlands" & 3444 & 15.5 & 222.19 & 167 & 10.77 & 4.85 \\
\hline New Zealand* & 650 & 3.6 & 180.56 & 26 & 7.22 & 4.00 \\
\hline Nigeria & 1282 & 97.2 & 13.19 & 4 & 0.04 & 0.31 \\
\hline Norway" & 1105 & 4.4 & 251.14 & 22 & 5.00 & 1.99 \\
\hline Pakistan & 758 & 134.1 & 5.65 & 1 & 0.01 & 0.13 \\
\hline
\end{tabular}


Table 2 (cont.): Data on countries pertaining to economy, population and astronomers

\begin{tabular}{|c|c|c|c|c|c|c|}
\hline Country & $\begin{array}{l}\text { IMF quota } \\
\text { (SDR) }\end{array}$ & $\begin{array}{c}\text { Population } \\
\text { (millions) }\end{array}$ & $\begin{array}{c}\text { Wealth } \\
\text { (SDR/million) }\end{array}$ & IAU members & $\begin{array}{c}\text { astronomers } \\
\text { (/million) }\end{array}$ & $\begin{array}{l}\text { astronomers } \\
(\text { SSDR }) \times 100\end{array}$ \\
\hline$\overline{\text { Paraguay }}$ & 72 & 5 & 14.40 & 1 & 0.20 & 1.39 \\
\hline Pen* & 466 & 23.9 & 19.50 & 1 & 0.04 & 0.21 \\
\hline Philippines & 633 & 71.5 & 8.85 & 1 & 0.01 & 0.16 \\
\hline Poland* & 989 & 38.6 & 25.62 & 117 & 3.03 & 11.83 \\
\hline Portugal* & 558 & 9.9 & 56.36 & 17 & 1.72 & 3.05 \\
\hline Romania* & 754 & 22.6 & 33.36 & 37 & 1.64 & 4.91 \\
\hline Russia* & 4313 & 147.1 & 29.32 & 344 & 2.34 & 7.98 \\
\hline S. Korea* & 800 & 46.4 & 17.24 & 51 & 1.10 & 6.38 \\
\hline Saudi Arabia* & 5131 & 18.8 & 272.93 & 11 & 0.59 & 0.21 \\
\hline Singapore & 358 & 3 & 119.33 & 2 & 0.67 & 0.56 \\
\hline Slovakia* & 257 & 5.4 & 47.59 & 27 & 5.00 & 10.51 \\
\hline Slovenia & 151 & 2 & 75.50 & 5 & 2.50 & 3.31 \\
\hline South Africa* & 1365 & 37.9 & 36.02 & 46 & 1.21 & 3.37 \\
\hline Spain ${ }^{\star}$ & 1935 & 39.3 & 49.24 & 204 & 5.19 & 10.54 \\
\hline Sri Lanka & 304 & 18.3 & 16.61 & 2 & 0.11 & 0.66 \\
\hline Sweden ${ }^{*}$ & 1614 & 8.8 & 183.41 & 95 & 10.80 & 5.89 \\
\hline Switzerland* & 2470 & 7.1 & 347.89 & 70 & 9.86 & 2.83 \\
\hline Tajikistan* & 60 & 5.8 & 10.17 & 7 & 1.19 & 11.67 \\
\hline Thailand & 574 & 60.8 & 9.44 & 3 & 0.05 & 0.52 \\
\hline Turkey & 642 & 63.7 & 10.08 & 53 & 0.83 & 8.26 \\
\hline UK * & 7415 & 60.5 & 122.56 & 550 & 9.09 & 7.42 \\
\hline Ukraine* & 997 & 50.5 & 19.74 & 119 & 2.36 & 11.94 \\
\hline Uruguay* & 225 & 3.2 & 70.31 & 5 & 1.56 & 2.22 \\
\hline USA* & 26527 & 267.6 & 99.13 & 2235 & 8.35 & 8.43 \\
\hline Uzbekistan & 200 & 22.5 & 8.89 & 8 & 0.36 & 4.00 \\
\hline Vatican City & & & & 5 & & \\
\hline Venezuela* & 1951 & 22.3 & 87.49 & 9 & 0.40 & 0.46 \\
\hline Vietnam & 242 & 75.4 & 3.21 & 2 & 0.03 & 0.83 \\
\hline Yugoslavia & & 10.5 & 0.00 & 25 & 2.38 & \\
\hline
\end{tabular}

- IAU member 


\section{Conclusions}

In conclusion I note that I have not tried to correlate the astronomical research index (ARI), which measures research potential based on the prevailing conditions in a country, with IAU astronomers/million of population, which is a rough measure of actual astronomical activity. If that is a useful exercise, then astronomers from all these countries should first try to determine their ARI values, which requires careful assessment and local knowledge.

The potential benefits of such an exercise would be a more ready identification of the true weaknesses and impediments in each country's overall astronomical research effort.

\section{References}

IAU Information Bull. 82, 1998 (14, June).

Europa World Year Book, 1998, Europa Publications Ltd, London.

Martinez, P. 2001, this volume, pp. 221-229.

\section{Discussion}

Sterken suggested that in some countries with high per capita incomes there are several small astronomy research groups in small universities. Except for criterion 15 (national scientific culture), to which Hearnshaw had given the lowest weight, the ARI for such institutes (as opposed to the nation as a whole) would put those institutes below the level at which Hearnshaw considered effective research to be possible. Sterken expressed concern that, if governments were to apply the ARI to individual institutes, they might be led to wrong conclusions about funding. Hearnshaw expressed doubt that there were insitutuions in developed countries lacking the essential funding, resources and facilities for research and yet producing useful results. If, however, he was wrong about that, he believed that publication of his criteria was as likely to lead governments to increase the resources of such institutions as the reverse.

Martinez suggested that good communications (criterion 10) should rank higher than Hearnshaw had suggested - on a par with, or just below, access to good computers (criterion 2). Good communications would automatically take care of a number of Hearnshaw's other criteria (3: library access, 5: critical mass of researchers, 6: telescope or data access and 7: a teaching program). Hearnshaw agreed that good communications are vital and that the ordering of his criteria is partly subjective. He had no quarrel with Martinez' ordering but pointed out that, before about 1950, astronomers could communiucate only by ordinary mail and yet did much good research. 